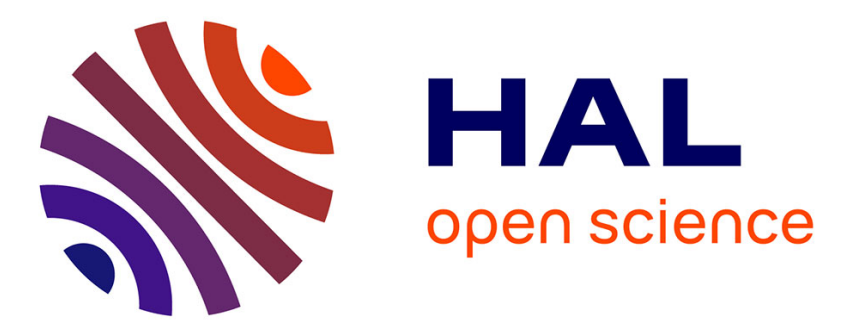

\title{
Off-line compensation of the tool path deviations on robotic machining: Application to incremental sheet forming
}

Jérémy Belchior, Mario Guillo, Eric Courteille, Patrick Maurine, Lionel Leotoing, Dominique Guines

\section{To cite this version:}

Jérémy Belchior, Mario Guillo, Eric Courteille, Patrick Maurine, Lionel Leotoing, et al.. Offline compensation of the tool path deviations on robotic machining: Application to incremental sheet forming. Robotics and Computer-Integrated Manufacturing, 2013, 29 (4), pp.58-69. 10.1016/j.rcim.2012.10.008 . hal-00926215

\section{HAL Id: hal-00926215 \\ https://hal.science/hal-00926215}

Submitted on 9 Jan 2014

HAL is a multi-disciplinary open access archive for the deposit and dissemination of scientific research documents, whether they are published or not. The documents may come from teaching and research institutions in France or abroad, or from public or private research centers.
L'archive ouverte pluridisciplinaire HAL, est destinée au dépôt et à la diffusion de documents scientifiques de niveau recherche, publiés ou non, émanant des établissements d'enseignement et de recherche français ou étrangers, des laboratoires publics ou privés. 


\title{
Off-line compensation of the tool path deviations on robotic machining: Application to Incremental Sheet Forming
}

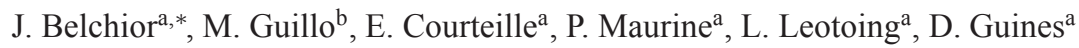 \\ ${ }^{a}$ Université Européenne de Bretagne, INSA-LGCGM EA-3913, 20 Avenue des Buttes de Coësmes, 35043, \\ Rennes Cedex, France \\ ${ }^{b}$ Institut Maupertuis, Contour Antoine de St-Exupéry Campus de Ker Lann, 35170, Bruz, France
}

\begin{abstract}
In this paper, a coupling methodology is involved and improved to correct the tool path deviations induced by the compliance of industrial robots during an incremental sheet forming task. For that purpose, a robust and systematic method is first proposed to derive the elastic model of their structure and an efficient FE simulation of the process is then used to predict accurately the forming forces. Their values are then defined as the inputs of the proposed elastic model to calculate the robot TCP pose errors induced by the elastic deformations. This avoid thus a first step of measurement of the forces required to form a test part with a stiff machine. An intensive experimental investigation is performed by forming a classical frustum cone and a non-symetrical twisted pyramid. It validates the robustness of both the FE analysis and the proposed elastic modeling allowing the final geometry of the formed parts to converge towards their nominal specifications in a context of prototyping applications.
\end{abstract}

Keywords: robot machining, elastic modeling, robot calibration, stiffness identification, off-line compensation, incremental sheet forming

\section{Introduction}

In order to reduce manufacturing costs and to improve production flexibility, the industrial robot manipulators are nowadays involved for processes such as machining, assembly or forming [1], [2]. Robots can be used for Incremental Sheet Forming (ISF) which is an interesting process for small series production and prototyping [3]. In ISF the sheet is deformed locally by successive paths of a simple tool. It means that lower forming forces than stamping are needed to form a part. These forming forces are fundamental data to predict the tool pose deviations of the robot [1]. These deviations are mainly due to the elastic deformations of the robot structure which lack of stiffness in comparison to dedicated machines [4], [5]. The resulting Tool Center Point (TCP) pose

\footnotetext{
the present work is supported in part by the European Union (EU). EU is committed in Brittany via FEDER founds.

* Corresponding author

Email addresses: jeremy.belchior@insa-rennes.fr(J. Belchior),

mario.guillo@institutmaupertuis.fr(M. Guillo),

eric.courteille@insa-rennes.fr(E.Courteille),patrick.maurine@insa-rennes.fr (P. Maurine), lionel.leotoing@insa-rennes.fr(L. Leotoing),

dominique.guines@insa-rennes.fr(D. Guines)
} 
errors degrade the process results in terms of geometry, surface, etc. In past decades, much of the work in the area of robot calibration including studies on the modeling of their structure, the measurement data collection and the model error identification has been done [6], [7], [8]. For that purpose two main approaches are available in the literature.

The first approach is to perform the dynamic elastic modeling of the robot structure in order to compensate by a linear or non linear feedback control the elastic deformations of the structure that degrade the TCP pose accuracy [9], [10], [11]. Outputs of such control consist in modifying the actuator torques. Therefore its implementation is difficult in actual industrial robots where only the TCP pose is controlled [12]. Moreover, the dynamic parameters (inertia, center of gravity, gear ratio) must be identified by dedicated methodologies [8], [13].

The second approach is based on realistic parametric models of the robots to predict the elastic deformations. The methodologies proposed in the literature are based either on Lumped-parameter [14], [15], [16] or more realistic Finite Element models [17], [18], [19]. Since outputs of these models are TCP pose errors, the term elastic model is used. As a result, a correction of the tool path deviations in the programming language of the controller (real-time or off-line programming) is possible. This method has already been applied on a Two Point Incremental Forming (TPIF) process (a supporting tool is used to hold the sheet on the backside) [20], [1]. In these works, the tool path deviations are computed with a Multy Body System modeling (MBS) of the robot structure coupled to a Finite Element analysis (FE). In the MBS model, the links are assumed rigid and the elastic behavior of the robot structure is described considering the joint stiffness only. The ISF FE simulation computes the estimated forming forces required to form the part assuming an ideal stiff robot. These values are then used to estimate with the MBS model the TCP pose errors that are due to the robot elastic deformations.

The main objective of our paper is to bring consistent contributions to these last works. For that purpose, our work focussed on the following points:

1. The TCP pose errors induced by the elastic deformations are calculated with a parametrical modeling method based on a new notation instead of a MBS modeling. The main advantage of this approach is that a realistic and complete $3 \mathrm{D}$ elastic model can be derived automatically for any industrial robot manipulators including openand closed-loop structures. Thereby the robot structure can be described considering the joint and the link stiffness.

2. Thanks to an efficient FE simulation of the process, the predicted forming forces are calculated and then used as inputs of the proposed elastic model. The advantage of this approach is to avoid the measuring of the forming forces during a first run without any compensations as in [20]. Actually in the case of really compliant robots, the measuring forces might be really lower as those exerted by an assuming stiff structure and this can lead to inaccurate corrections of the tool path.

3. In order to validate the coupling approach an intensive experimental investigation is performed by considering on one hand a classical frustum cone as in [20] and on the other hand a twisted pyramid. The non-symetrical geometry of this last one allows to validate the robustness of both the FE analysis and the elasto geometricalmodeling.

The paper is organized as follows. First the ISF process requirements are given and the TCP pose accuracy abilities of a FANUC S420iF are verified according to 
ISO-9283 standard. Next sections describe respectively the new proposed systematic elastic modeling and its application to the FANUC S420iF. The resulting elastic model of its structure is next identified and then involved to compensate both the geometrical errors and elastic deformations during the ISF of a frustum cone and a twisted pyramid on an aluminium sheet. For each shape, experimental results are deeply analyzed and discussed.

\section{Incremental Forming process requirements}

To be as efficient as dedicated machines the serial robots have to verify the process requirements. For example, to form a frustum cone of $40 \mathrm{~mm}$ depth and $50^{\circ}$ wall angle with a $1.2 \mathrm{~mm}$ thick aluminium sheet, a maximum force of $600 \mathrm{~N}$ is needed. Feed rates usually programmed are included between $1 \mathrm{~m} / \mathrm{min}$ and $2 \mathrm{~m} / \mathrm{min}$ so the process can be considered as quasi-static [21]. The forces required to form thin aluminium parts are compatible with the FANUC robot S420iF. It is a typical robot used for mechanical assembly with six degrees of freedom and a payload capacity of $1200 \mathrm{~N}$. It has a kinematic closed loop that increases the global stiffness of the structure. In order to verify the robot capabilities versus the process, a diagnostic of the robot has been made according the ISO-9283 standard [22]. Results obtained with ROBOSCOPE ${ }^{\mathbb{C}}$ software and the Nikon Metrology K600-10 photogrammetric measurement system are presented in TABLE 1 . The system has a pose measuring accuracy up to $37 \mu \mathrm{m}$ for a single point. As one can see in TABLE 1, the maximum compliance and accuracy errors respectively of 3.3 and $1.6 \mathrm{~mm}$ clearly show that this robot cannot achieve ISF parts with an acceptable level of accuracy $( \pm 0.5 \mathrm{~mm}$ [23]). Therefore an efficient off-line compensation of the robot tool path deviations has to be performed.

Table 1: Certifications's results based on ISO-9283 standard

\begin{tabular}{ccc}
\hline & Position $(\mathrm{mm})$ & Orientation $(\mathrm{mdeg})$ \\
\hline Mean Repeatability error & 0.134 & 15.271 \\
\hline Max Repeatability error & 0.176 & 20.059 \\
\hline Mean Accuracy error & 0.914 & 137.239 \\
\hline Max Accuracy error & 1.644 & 165.346 \\
\hline Max Compliance error $(650 \mathrm{~N})$ & 3.253 & - \\
\hline
\end{tabular}

\section{Elastic modeling}

Denavit-Hartenberg or Khalil-Kleinfinger notations are usually used for the geometrical modeling of industrial robots [6], [8]. Finite Element theory [24] is involved to derive the elastic model by discretizing the robot structure into a set of nodes and beams. Nodes can represent the start or the end of a link, an intermediate frame or a characteristic point on the real structure. However, the definition of the frames used for the geometrical modeling is not really appropriated for the elastic modeling one [17] since in the classical beam theory $\mathbf{x}$ axis has to be along the neutral axis [24]. In this paper, the definition of a new parameter table is proposed to make mutually coherent the notation of both geometrical and elastic models in order to describe with a minimum set of parameters open- (Figure 1) and closed-loop structure robots (Figure 2). For that purpose, the systematic modeling is performed by the definition of two specific frames: 
- Joint frames $R_{j}$ : Khalil-Kleinfinger notation is used and allows to have the geometrical situation of frames $R_{j}$ in the base frame $R_{0}$.

- Link frames $R_{u, v}$ : links can be considered either rigid nor deformable. If a link is considered deformable, the classical beam theory is then used to described its mechanical behaviour. $\mathbf{x}$ axis has to be along the neutral axis [24]. A notation based on three parameters allows to have the geometrical description of the link frame $R_{u, v}$ in the base frame $R_{0}$.

\subsection{Open-loop robots}

The system is composed of $n$ joints and $n+1$ links. The link $C_{0}$ is the base of the robot and $C_{n}$ the link holding the tool.

\subsubsection{Joint frames $R_{j}$}

Joint $j$ defined between two nodes $l$ and $u$, connects link $C_{j-1}$ with link $C_{j} . C_{j-1}$ is the precedent link of $C_{j}$. The frame $R_{j}$, which is fixed with link $C_{j}$ and connected to the node $l$, is defined such that:

- $\mathbf{z}_{j}$ is along the axis of joint $j$.

- $\mathbf{x}_{j}$ is taken along the common normal between $\mathbf{z}_{j}$ and $\mathbf{z}_{j+1}$. If $\mathbf{z}_{j}$ and $\mathbf{z}_{j+1}$ are parallel, the choice of $\mathbf{x}_{j}$ is not unique. In order to minimize the number of parameters, $\mathbf{x}_{j}$ will be placed if possible along the neutral axis of the $\operatorname{link} C_{j}$.

\subsubsection{Link frames $R_{u, v}$}

It is necessary to define a specific frame when a link $C_{j}$ is considered deformable. This is defined between two nodes $u$ and $v$. A link frame $R_{u, v}$ is associated to the node $u$ and defined such that:

- $\mathbf{x}_{u, v}$ is along the neutral axis of the link $C_{j}$.

- $\mathbf{z}_{u, v}$ is along the main inertia axis of the link $C_{j}$.

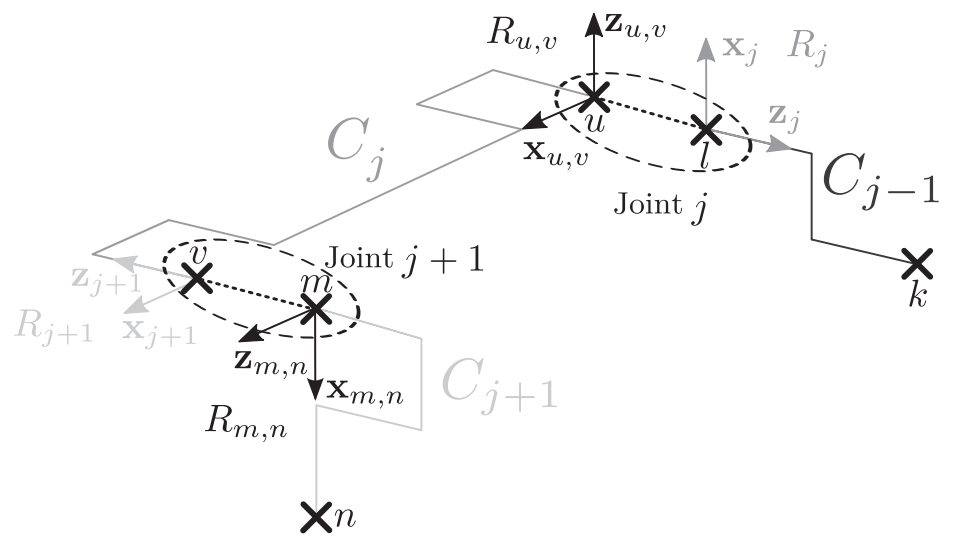

Figure 1: Modeling of open loop structures 


\subsection{Closed-loop structure robots}

The system is composed of $L$ joints and $n+1$ links, where $C_{0}$ is the base and $L>n$. The geometric description of a structure with closed-loops is defined by an equivalent tree structure obtained by cutting each closed loop at one of its joints and by adding two frames at each cut joint. The $(L \times 1)$ joint variable vector is written $\mathbf{q}=\left[\begin{array}{lll}\mathbf{q}_{a} & \mathbf{q}_{p} & \mathbf{q}_{c}\end{array}\right]^{T}$ with:

- $\mathbf{q}_{a}$ : the vector containing the $N$ active joint variables.

- $\mathbf{q}_{p}$ : the vector containing the $p=n-N$ passive joint variables of the equivalent tree structure.

- $\mathbf{q}_{c}$ : the vector containing the $B$ variables of the cut joints.

\subsubsection{Joint frames $R_{j}$}

Joint $j$ defined between two nodes $v$ and $m$, connects link $C_{i=a(j)}$ with link $C_{j}$. $C_{i}$ is the precedent link of $C_{j}$. The topology of the system is defined by $a(j)$ for $j=1, \ldots, n$. The frame $R_{i}$, which is fixed with link $C_{i}$ and connected to the node $l$, is defined such that:

- $\mathbf{z}_{i}$ is along the axis of joint $i$.

- $\mathbf{x}_{i}$ is taken along the common normal between $\mathbf{z}_{i}$ and $\mathbf{z}$ of one of the links held by link $C_{i}$.

- If the link $C_{i}$ holds more than one link, for example $C_{j}$ and $C_{k}$, it is necessary to define two axis:

$\mathbf{x}_{i}$ which is the common normal between $\mathbf{z}_{i}$ and $\mathbf{z}_{k}$

$\mathbf{x}_{i}^{\prime}$ which is the common normal between $\mathbf{z}_{i}$ and $\mathbf{z}_{j}$

\subsubsection{Link frames $R_{u, v}$}

It is necessary to define a specific frame when a link $C_{i}$ is considered deformable. If the link $C_{i}$ is composed by two nodes, the link frame $R_{u, v}$ is defined according to open-loop robot methodology. If a link $C_{i}$ is composed by more than two nodes, it is necessary to define a new indice $w . C_{i}^{(w)}$ with $w=1, \ldots, p$ defines a sub-link of $C_{i}$. It is necessary to define a link frame $R_{u, v}$ for each sub-link $C_{i}^{(w)}$ at the node $u$ which is a common node of $C_{i}^{(w)}$ links. The link frame $R_{u, v}$ is defined such that:

- $\mathbf{x}_{u, v}$ is along the sub-link $C_{i}^{(w)}$.

- $\mathbf{z}_{u, v}$ is along the main inertia axis of the sub-link $C_{i}^{(w)}$.

\subsection{Description of the structure in the base frame $R_{0}$}

In order to perform stiffness matrix assembly it is necessary to define the geometrical situation of the joint frame $R_{j}$ and the link frame $R_{u, v}$ in $R_{0}$ [24]. 


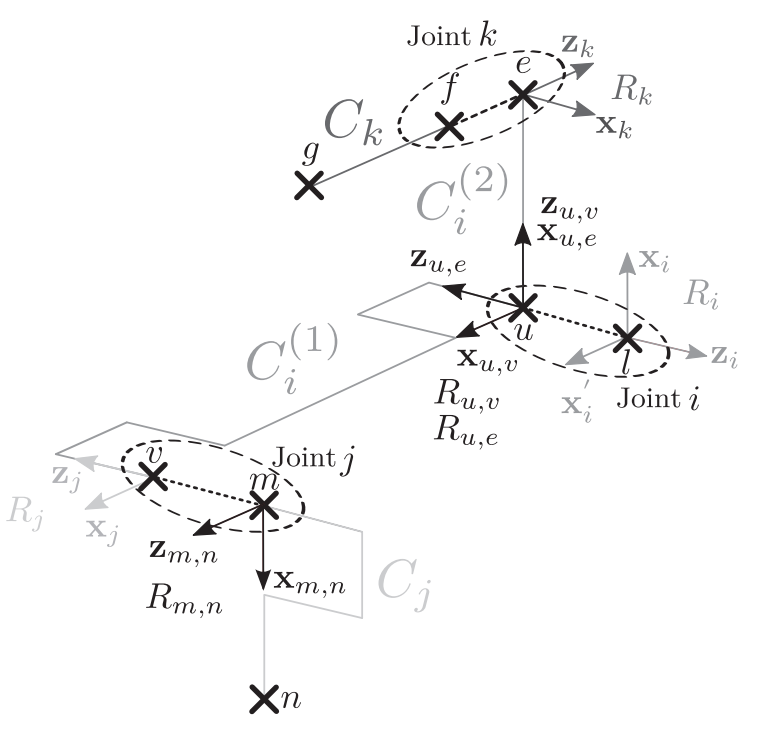

Figure 2: Modeling of closed loop structures

\subsubsection{Joint frames $R_{j}$}

The situation of $R_{j}$ in the frame $R_{i}$, requires six parameters $\gamma_{j}, b_{j}, \alpha_{j}, d_{j}, \theta_{j}, r_{j}$. The definition of these parameters is available in [25]. The homogeneous transformation matrix ${ }^{i} \mathbf{T}_{j}$ linking $R_{j}$ to $R_{i}$ is calculated by:

$$
\begin{aligned}
{ }^{i} \mathbf{T}_{j}= & \operatorname{Rot}\left(\mathbf{z}, \gamma_{j}\right) \operatorname{Trans}\left(\mathbf{z}, b_{j}\right) \operatorname{Rot}\left(\mathbf{x}, \alpha_{j}\right) \\
& \operatorname{Trans}\left(\mathbf{x}, d_{j}\right) \operatorname{Rot}\left(\mathbf{z}, \theta_{j}\right) \operatorname{Trans}\left(\mathbf{z}, r_{j}\right) \\
= & {\left[\begin{array}{ccc|c}
{ }^{i} \mathbf{A}_{j} & { }^{i} \mathbf{P}_{j} \\
\hline 0 & 0 & 0 & 1
\end{array}\right]_{(4 \times 4)} . }
\end{aligned}
$$

The matrix ${ }^{i} \mathbf{A}_{j}$ gives the orientation of the frame $R_{j}$ with respect to $R_{i}$. The transformation ${ }^{0} \mathbf{T}_{j}$, which represents the situation of the frame $R_{j}$ with respect to $R_{0}$, is obtained by post-multiplication of matrices ${ }^{i} \mathbf{T}_{j}$.

\subsubsection{Link frames $R_{u, v}$}

The description of the orientation of $R_{u, v}$ in the frame $R_{j}$, requires three parameters $\delta_{j}, \eta_{j}$ and $\kappa_{j}$.

- $\delta_{j}$ : angle of rotation around $\mathbf{x}_{j}$ axis.

- $\eta_{j}$ : angle of rotation around $\mathbf{y}_{j}$ axis.

- $\kappa_{j}$ : angle of rotation around $\mathbf{z}_{j}$ axis.

A parameter $\chi_{j}$ is introduced such that:

- $\chi_{j}=0$ if the link $C_{j}$ is considered as rigid.

- $\chi_{j}=1$ if the link $C_{j}$ is considered as deformable. 
tion:

The description of the situation of $R_{u, v}$ in the joint frame $R_{j}$ is given by the rela-

$$
{ }^{j} \mathbf{T}_{u, v}=\boldsymbol{\operatorname { R o t }}\left(\mathbf{x}, \delta_{j}\right) \boldsymbol{\operatorname { R o t }}\left(\mathbf{y}, \eta_{j}\right) \boldsymbol{\operatorname { R o t }}\left(\mathbf{z}, \kappa_{j}\right) .
$$

Then the description of the situation of $R_{u, v}$ in the base frame $R_{0}$ is described by the following relation:

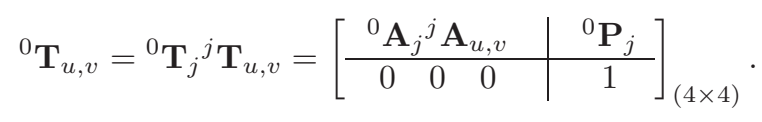

\subsection{Elastic model}

\subsubsection{Beam element}

A beam is defined by two nodes and used when a link is considered as deformable. The displacements of a node can be calculated when a wrench is applied on it. The vector of nodal wrench that is applied at node $v$ is:

$$
\mathbf{F}_{v}=\left[\begin{array}{ll}
f_{v} & m_{v}
\end{array}\right]^{T}
$$

with:

- $\mathbf{f}_{v}=\left[\begin{array}{lll}f_{v, x} & f_{v, y} & f_{v, z}\end{array}\right]^{T}$ the force applied at node $v$.

- $\mathbf{m}_{v}=\left[\begin{array}{lll}m_{v, x} & m_{v, y} & m_{v, z}\end{array}\right]^{T}$ the torque.

Expressed in $R_{u, v}$ and $R_{0}$, the wrench $\mathbf{F}_{v}$ will be written respectively $\mathbf{F}_{v}$ and ${ }^{0} \mathbf{F}_{v}$. $R_{u, v}$ is the local frame attached to the beam defined between $u$ and $v$ with $\mathbf{x}_{u, v}$ along the line $(u, v)$ (Figure 3). The nodal displacement resulting of the application of $\mathbf{F}_{v}$ at the node $v$ is:

$$
\Delta \mathbf{X}_{v}=\left[\begin{array}{cc}
\Delta \mathbf{P}_{v} & \Delta \Phi_{v}
\end{array}\right]^{T}
$$

with:

- $\Delta \mathbf{P}_{v}=\left[\begin{array}{ccc}\Delta P_{v, x} & \Delta P_{v, y} & \Delta P_{v, z}\end{array}\right]^{T}$ is the translational displacement vector of $v$.

- $\Delta \Phi_{v}=\left[\begin{array}{ccc}\Delta \phi_{v, x} & \Delta \phi_{v, y} & \Delta \phi_{v, z}\end{array}\right]^{T}$ the vector gathering the three rotation angles of the beam section.

\subsubsection{Stiffness matrix of flexible joints}

In the local frame, the stiffness of the compliant joint defined between the nodes $v$ and $m$ and whose axis is along $\mathbf{z}_{j}$ of the frame $R_{j}$ is described by the $(12 \times 12)$ stiffness matrix:

$$
\mathbf{K}_{j}=\left[\begin{array}{c|c}
\mathbf{K}_{j}^{D} & -\mathbf{K}_{j}^{D} \\
\hline-\mathbf{K}_{j}^{D} & \mathbf{K}_{j}^{D}
\end{array}\right]_{(12 \times 12)}
$$

where: $\mathbf{K}_{j}^{D}=\operatorname{diag}\left[K_{r}, K_{r}, K_{a}, K_{r r}, K_{r r}, K_{a r}\right]$.

$K_{a}$ and $K_{r}$ are respectively the translational axial and radial stiffness. $K_{a r}$ and $K_{r r}$ are the rotational axial and radial stiffness [26]. 


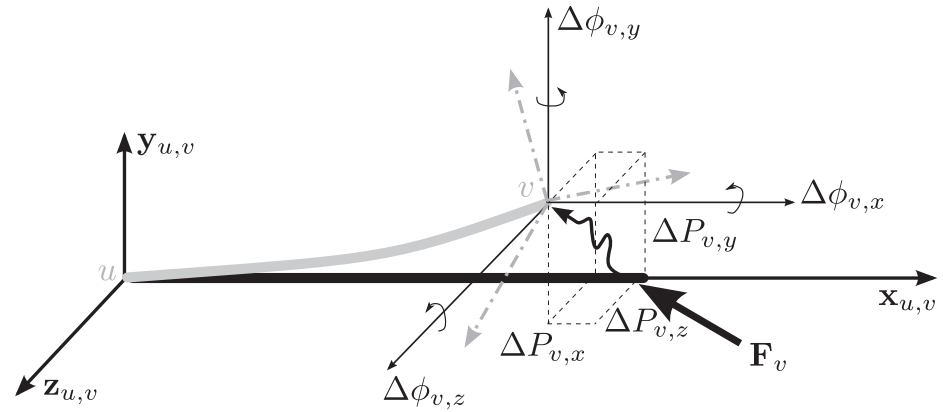

Figure 3: Elastic beam, nodal wrench and displacement

The calculation of the stiffness matrix components in $R_{0}$ is done by using the transformation matrix ${ }^{0} \mathbf{G}_{j}$ according to:

$$
{ }^{0} \mathbf{K}_{j}=\left({ }^{0} \mathbf{G}_{j}\right) \mathbf{K}_{j}\left({ }^{0} \mathbf{G}_{j}\right)^{-1}
$$

${ }^{0} \mathbf{G}_{j}$ is given by:

$$
{ }^{0} \mathbf{G}_{j}=\left[\begin{array}{llll}
{ }^{0} \mathbf{A}_{j} & \mathbf{0}_{3,3} & \mathbf{0}_{3,3} & \mathbf{0}_{3,3} \\
\mathbf{0}_{3,3} & \mathbf{A}_{j} & \mathbf{0}_{3,3} & \mathbf{0}_{3,3} \\
\mathbf{0}_{3,3} & \mathbf{0}_{3,3} & { }^{0} \mathbf{A}_{j} & \mathbf{0}_{3,3} \\
\mathbf{0}_{3,3} & \mathbf{0}_{3,3} & \mathbf{0}_{3,3} & { }^{0} \mathbf{A}_{j}
\end{array}\right]_{(12 \times 12)}
$$

\subsubsection{Stiffness matrix of beams}

For a beam, the stiffness matrix depends on its geometrical and mechanical parameters (TABLE 2). For a deformable link $C_{j}$, the stiffness matrix is the well-known 12 dimensional square matrix $\mathbf{K}_{u, v}$ defined into its local coordinate system $R_{u, v}$, it can be expressed by using four sub-matrices:

$$
\mathbf{K}_{u, v}=\left[\begin{array}{l|l}
\mathbf{K}_{u, v}^{11} & \mathbf{K}_{u, v}^{12} \\
\hline \mathbf{K}_{u, v}^{21} & \mathbf{K}_{u, v}^{22}
\end{array}\right]_{(12 \times 12)} .
$$

For which if the shear effects are neglected $\mathbf{K}_{u, v}^{11}, \mathbf{K}_{u, v}^{12}, \mathbf{K}_{u, v}^{21}$, et $\mathbf{K}_{u, v}^{22}$ are defined in the literature [24]. The calculation in $R_{0}$ of the stiffness matrix components is done by using the transformation matrix ${ }^{0} \mathbf{B}_{u, v}$ according to the relation:

$$
{ }^{0} \mathbf{K}_{u, v}=\left({ }^{0} \mathbf{B}_{u, v}\right) \mathbf{K}_{u, v}\left({ }^{0} \mathbf{B}_{u, v}\right)^{-1} .
$$

${ }^{0} \mathbf{B}_{u, v}$ is given by:

$$
{ }^{0} \mathbf{B}_{u, v}=\left[\begin{array}{cccc}
{ }^{0} \mathbf{A}_{j}{ }^{j} \mathbf{A}_{u, v} & \mathbf{0}_{3,3} & \mathbf{0}_{3,3} & \mathbf{0}_{3,3} \\
\mathbf{0}_{3,3} & { }^{0} \mathbf{A}_{j}{ }^{j} \mathbf{A}_{u, v} & \mathbf{0}_{3,3} & \mathbf{0}_{3,3} \\
\mathbf{0}_{3,3} & \mathbf{0}_{3,3} & { }^{0} \mathbf{A}_{j}{ }^{j} \mathbf{A}_{u, v} & \mathbf{0}_{3,3} \\
\mathbf{0}_{3,3} & \mathbf{0}_{3,3} & \mathbf{0}_{3,3} & { }^{0} \mathbf{A}_{j}{ }^{j} \mathbf{A}_{u, v}
\end{array}\right]_{(12 \times 12)}
$$

\subsubsection{Assembly technique}

The assembly of all matrices is then performed by adding the elastic effects at all nodes of the structure according to their indexes. It leads to the whole structure stiffness matrix ${ }^{0} \mathbf{K}_{c}$. The dimension of ${ }^{0} \mathbf{K}_{c}$ is $(6 H \times 6 H)$, where $H$ is the number of nodes. Then, boundary conditions are introduced. So the size of the stiffness matrix ${ }^{0} \mathbf{K}_{c}$ is 
Table 2: Geometrical and Mechanical parameters

\begin{tabular}{ccc}
\hline Geometrical parameters & Length \& Cross-sectional area & $L \& S$ \\
\hline \multirow{2}{*}{ Mechanical parameters } & $\begin{array}{c}\text { Young's \& Coulomb's modulus } \\
\text { Quadratic \& Polar moments }\end{array}$ & $\begin{array}{c}E \& G \\
I_{y}, I_{z} \& J\end{array}$ \\
\hline
\end{tabular}

reduced by deleting the rows and the columns corresponding to the nodes for which displacements are null. It leads to the ${ }^{0} \mathbf{K}_{c}^{*}$ stiffness matrix.

\subsubsection{Rigid link motion}

Before establishing relation between forces/displacements, it is necessary to include rigid link motion when a link is considered as rigid. Thus, the matrix ${ }^{0} \mathbf{A}_{c}^{*}$ includes the $M=6 \times\left(n-\sum \chi_{j}\right)$ relations of rigid link motion and boundary conditions. Finally rigid link motions can be expressed by the following relation:

$$
{ }^{0} \mathbf{A}_{c}^{* 0} \Delta \mathbf{X}_{c}^{*}=\mathbf{0}_{M, 1} .
$$

\subsubsection{Energy study}

It is possible to calculate the node displacements ${ }^{0} \Delta \mathbf{X}_{c}^{*}$, that verify the relation ${ }^{0} \mathbf{F}_{c}^{*}={ }^{0} \mathbf{K}_{c}^{* 0} \Delta \mathbf{X}_{c}^{*}$ under the constrains given by the relation (12). This problem can be solved by searching the extremum conditions of the potential energy [24]. A linear system is then obtained:

$$
\left[\begin{array}{c}
{ }^{0} \mathbf{F}_{c}^{*} \\
\mathbf{0}_{M, 1}
\end{array}\right]=\left[\begin{array}{cc}
{ }^{0} \mathbf{K}_{c}^{*} & { }^{0} \mathbf{A}_{c}^{* T} \\
{ }^{0} \mathbf{A}_{c}^{*} & \mathbf{0}_{M, M}
\end{array}\right]\left[\begin{array}{c}
{ }^{0} \Delta \mathbf{X}_{c}^{*} \\
\lambda_{M}
\end{array}\right]
$$

where $\lambda_{M}$ are the Lagrange multipliers. By defining:

$$
{ }^{0} \mathbf{F}_{G}=\left[\begin{array}{c}
{ }^{0} \mathbf{F}_{c}^{*} \\
\mathbf{0}_{M, 1}
\end{array}\right] \quad{ }^{0} \mathbf{K}_{G}=\left[\begin{array}{cc}
{ }^{0} \mathbf{K}_{c}^{*} & { }^{0} \mathbf{A}_{c}^{* T} \\
{ }^{0} \mathbf{A}_{c}^{*} & \mathbf{0}_{M, M}
\end{array}\right] \quad{ }^{0} \Delta \mathbf{X}_{G}=\left[\begin{array}{c}
{ }^{0} \Delta \mathbf{X}_{c}^{*} \\
\lambda_{M, 1}
\end{array}\right]
$$

The relation (13) becomes:

$$
{ }^{0} \mathbf{F}_{G}={ }^{0} \mathbf{K}_{G}{ }^{0} \Delta \mathbf{X}_{G} .
$$

Since the relation (14) includes the boundary conditions and the rigid link motions, it is symmetric, positive definite and invertible. The node displacements are given by the relation:

$$
{ }^{0} \Delta \mathbf{X}_{G}=\left({ }^{0} \mathbf{K}_{G}\right){ }^{-1}{ }^{0} \mathbf{F}_{G} .
$$

The solution vector ${ }^{0} \Delta \mathbf{X}_{G}$ contains the values of all node displacements and Lagrange multipliers.

\subsubsection{Equivalent structure}

For a given load on the structure (link own weight, payload, external forces, etc.), it is possible to calculate the elastic displacements ${ }^{0} \Delta \mathbf{X}_{E}$ at the node corresponding to the TCP. For that purpose, the whole elastic behavior of the structure is described by an equivalent stiffness matrix ${ }^{0} \mathbf{K}_{e q}$ and an equivalent wrench ${ }^{0} \mathbf{F}_{e q}$. In the general case, the stiffness matrix and the vector of nodal wrenches and displacements are defined between the base and the TCP nodes by using the following partition matrices: 


$$
\left[\begin{array}{c}
{ }^{0} \mathbf{F}_{R} \\
\hline{ }^{0} \mathbf{F}_{E}
\end{array}\right]=\left[\begin{array}{c|c}
{ }^{0} \mathbf{K}_{R, R} & { }^{0} \mathbf{K}_{R, E} \\
\hline{ }^{0} \mathbf{K}_{E, R} & { }^{0} \mathbf{K}_{E, E}
\end{array}\right]\left[\begin{array}{c}
{ }^{0} \Delta \mathbf{X}_{R} \\
\hline{ }^{0} \Delta \mathbf{X}_{E}
\end{array}\right] .
$$

The indice $R$ corresponds to the components of all nodes excepted the node $E$ defining the TCP. Thus one can obtain:

$$
\left\{\begin{aligned}
{ }^{0} \mathbf{F}_{R} & ={ }^{0} \mathbf{K}_{R, R}{ }^{0} \Delta \mathbf{X}_{R}+{ }^{0} \mathbf{K}_{R, E}{ }^{0} \Delta \mathbf{X}_{E} \\
{ }^{0} \mathbf{F}_{E} & ={ }^{0} \mathbf{K}_{E, R}{ }^{0} \Delta \mathbf{X}_{R}+{ }^{0} \mathbf{K}_{E, E}{ }^{0} \Delta \mathbf{X}_{E}
\end{aligned}\right.
$$

The vector of nodal displacements ${ }^{0} \Delta \mathbf{X}_{E}$ can be calculated according to:

$$
{ }^{0} \Delta \mathbf{X}_{E}=\left({ }^{0} \mathbf{K}_{e q}\right){ }^{-1}{ }^{0} \mathbf{F}_{e q}
$$

with:

$$
\begin{aligned}
& { }^{0} \mathbf{F}_{e q}={ }^{0} \mathbf{F}_{E}-{ }^{0} \mathbf{K}_{E, R}\left({ }^{0} \mathbf{K}_{R, R}\right){ }^{-1}{ }^{0} \mathbf{F}_{R} \\
& { }^{0} \mathbf{K}_{e q}={ }^{0} \mathbf{K}_{E, E}-{ }^{0} \mathbf{K}_{E, R}\left({ }^{0} \mathbf{K}_{R, R}\right){ }^{-1}{ }^{0} \mathbf{K}_{R, E}
\end{aligned}
$$

The equation (18) defines the equivalent elastic model which predict the whole elastic behavior of the structure.

\subsubsection{Forward elastic Model}

The Forward Geometrical Model is defined by the following relation that links the controlled pose ${ }^{0} \mathbf{X}_{E}$ to the joint configuration $\mathbf{q}^{k}$ and the vector of the geometrical parameters $\xi$ :

$$
{ }^{0} \mathbf{X}_{E}=\left[\begin{array}{c}
{ }^{0} \mathbf{P}_{E} \\
{ }^{0} \Phi_{E}
\end{array}\right]=f\left(\mathbf{q}^{k}, \xi\right)
$$

where ${ }^{0} \mathbf{P}_{E}$ and ${ }^{0} \Phi_{E}$ give respectively the cartesian position and the orientation of the end-effector relative to $R_{0} .{ }^{0} \mathbf{X}_{E}$ is given by post multiplication of homogenous matrices according to (1). The Forward Elastic Model whose elastic parameters have to be identified can be described by the relation (21) for a joint configuration $\mathbf{q}^{k}$ :

$$
{ }^{0} \Delta \mathbf{X}_{E}=\left[\begin{array}{c}
{ }^{0} \Delta \mathbf{P}_{E} \\
{ }^{0} \Delta \Phi_{E}
\end{array}\right]=h\left(\mathbf{q}^{k}, \xi,{ }^{0} \mathbf{F}_{e q}, \Lambda, \Gamma\right)=\left({ }^{0} \mathbf{K}_{e q}\right){ }^{-1}{ }^{0} \mathbf{F}_{e q}
$$

where:

- $\xi$ : the geometrical parameters.

- ${ }^{0} \mathbf{F}_{e q}$ : the equivalent wrench defined in $R_{0}$.

- $\Lambda$ : the mechanical parameters of the beams.

- $\Gamma$ : the joint stiffness parameters.

The definition of the Forward Elastic Model is given by:

$$
{ }^{0} \check{\mathbf{X}}_{E}={ }^{0} \mathbf{X}_{E}+{ }^{0} \Delta \mathbf{X}_{E}
$$

Finally the elastic model is fully described by two parameter tables:

- The geometrical table for definition of joint frames $R_{j}$ in $R_{0}$.

- The geometrical table for definition of link frames $R_{u, v}$ in $R_{0}$. 


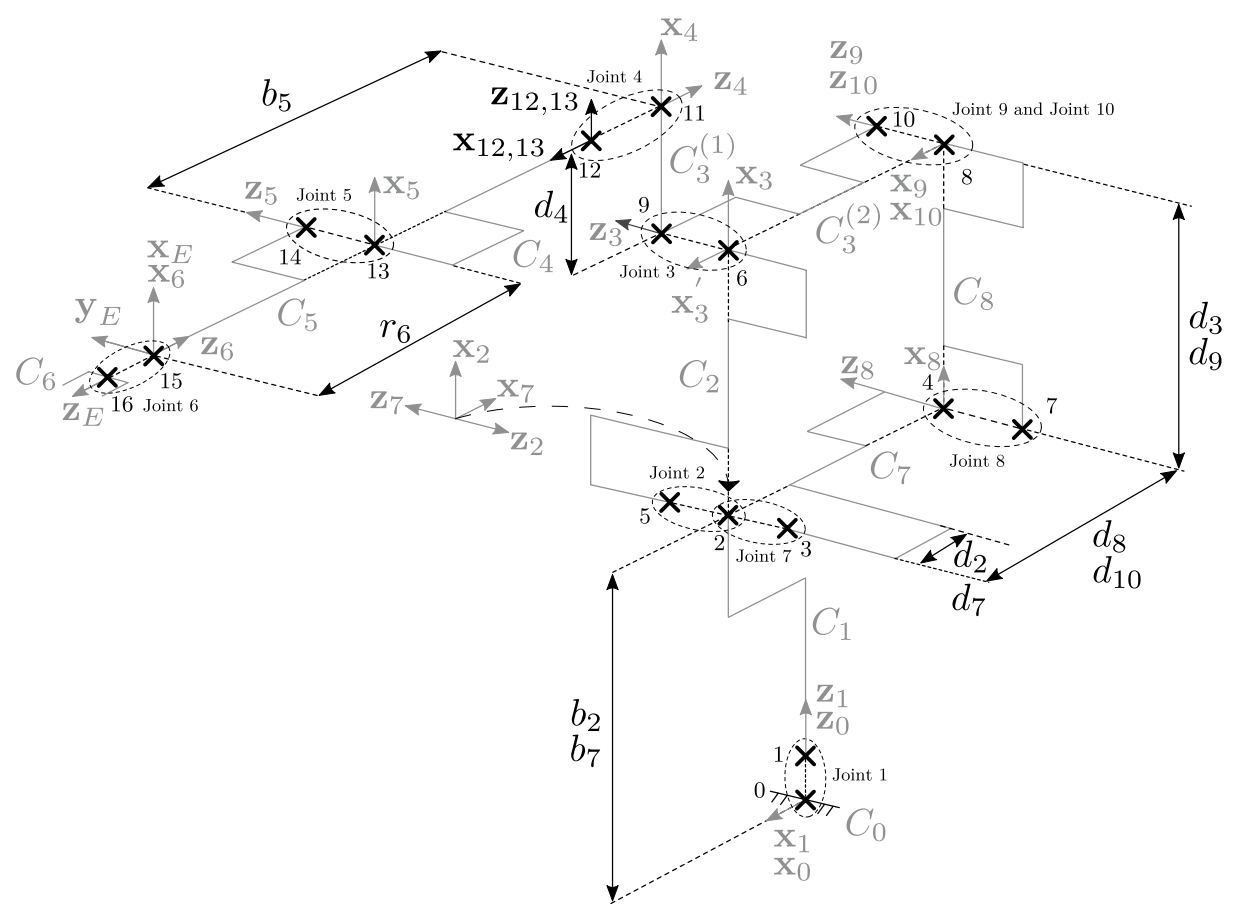

Figure 4: FANUC S420iF modeling

\section{Application to the modeling of a FANUC S420iF robot}

\subsection{Modeling}

The FANUC S420iF has a number $L=9$ revolute joints $\left(q_{j}=\theta_{j}\right)$ and $n+1=9$ links where link $C_{0}$ is the fixed base and $B=L-n=1$ closed loop. There are $N=6$ active joints. A complete segmentation of the structure can be done with 17 nodes (Figure 4). The vector $\mathbf{q}$ is defined by:

$$
\begin{gathered}
\mathbf{q}_{a}=\left[\begin{array}{llllll}
q_{1} & q_{2} & q_{4} & q_{5} & q_{6} & q_{7}
\end{array}\right]^{T}, \\
\mathbf{q}_{p}=\left[\begin{array}{ll}
q_{3} & q_{8}
\end{array}\right]^{T}, \quad \mathbf{q}_{c}=q_{9} .
\end{gathered}
$$

Closed-loop constraint gives : $q_{3}=q_{9}=-q_{8}=q_{2}+q_{7}$

\subsubsection{Joint frames $R_{j}$}

Joint frames are fixed according to the section 3.2.1. The geometrical table for joint frames $R_{j}$ of the FANUC S420iF is shown in Table 3. One can obtain ${ }^{0} \mathbf{X}_{E}={ }^{0} \mathbf{T}_{j}$ by post-multiplication matrices.

\subsubsection{Link frames $R_{u, v}$}

Link frames are fixed according to the section 3.2.2. For the FANUC S420iF, all links are assumed to be rigid except $C_{4}$ which ratio length/section is important. $C_{4}$ is then described as an hollow cylindrical beam made of steel. A link frame $R_{12,13}$ is fixed to the node 12 (Figure 5). Then it is possible to define the transformation ${ }^{4} \mathbf{T}_{12,13}$ 
Table 3: Geometrical table for the definition of the joint frames $R_{j}$

\begin{tabular}{ccccccccc}
\hline $\mathrm{j}$ & $\mathrm{a}(\mathrm{j})$ & $\mu_{j}$ & $\gamma_{j}$ & $b_{j}$ & $\alpha_{j}$ & $d_{j}$ & $\theta_{j}$ & $r_{j}$ \\
\hline 1 & 0 & 1 & 0 & 0 & 0 & 0 & $\theta_{1}$ & 0 \\
\hline 2 & 1 & 1 & 0 & $b_{2}$ & $-\frac{\pi}{2}$ & $d_{2}$ & $-\frac{\pi}{2}+\theta_{2}$ & 0 \\
\hline 3 & 2 & 0 & 0 & 0 & $\pi$ & $d_{3}$ & $\theta_{3}$ & 0 \\
\hline 4 & 3 & 1 & 0 & 0 & $-\frac{\pi}{2}$ & $d_{4}$ & $\theta_{4}$ & 0 \\
\hline 5 & 4 & 1 & 0 & $-b_{5}$ & $\frac{\pi}{2}$ & 0 & $\theta_{5}$ & 0 \\
\hline 6 & 5 & 1 & 0 & 0 & $-\frac{\pi}{2}$ & 0 & $\theta_{6}$ & $-r_{6}$ \\
\hline 7 & 1 & 1 & 0 & $b_{7}$ & $\frac{\pi}{2}$ & $d_{7}$ & $\pi+\theta_{7}$ & 0 \\
\hline 8 & 7 & 0 & 0 & 0 & 0 & $d_{8}$ & $-\frac{\pi}{2}+\theta_{8}$ & 0 \\
\hline 9 & 8 & 0 & 0 & 0 & 0 & $d_{9}$ & $-\frac{\pi}{2}+\theta_{9}$ & 0 \\
\hline 10 & 3 & 0 & $-\frac{\pi}{2}$ & 0 & 0 & $-d_{10}$ & 0 & 0 \\
\hline $\mathrm{E}$ & 6 & 0 & 0 & 0 & $\pi$ & 0 & 0 & 0 \\
\hline
\end{tabular}

with the three parameters $\delta_{4}, \eta_{4}$ and $\kappa_{4}$. There are more than one solution, but the most convenient is to choose the angle around $\mathbf{y}_{4}, \eta_{4}=\frac{\pi}{2}$. Equation (23) is obtained.

$$
{ }^{4} \mathbf{T}_{12,13}=\boldsymbol{\operatorname { R o t }}(\mathbf{x}, 0) \operatorname{Rot}\left(\mathbf{y}, \frac{\pi}{2}\right) \boldsymbol{\operatorname { R o t }}(\mathbf{z}, 0) .
$$

The description of all the links is shown in Table 4.

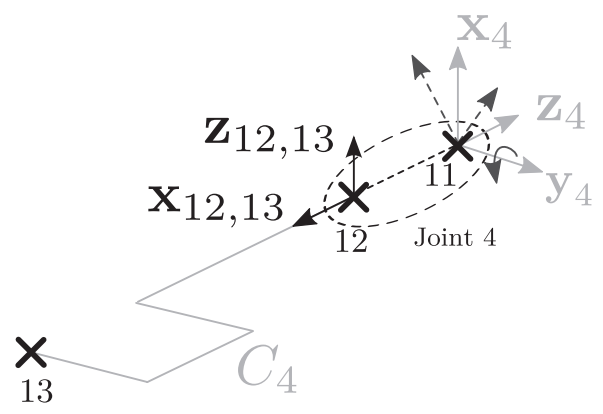

Figure 5: Transformation between joint frame $R_{4}$ and link frame $R_{12,13}$

Table 4: Geometrical table for link frames $R_{u, v}$ definition

\begin{tabular}{ccccccc}
\hline$C_{j}$ & $u$ & $v$ & $\chi_{j}$ & $\delta_{j}$ & $\eta_{j}$ & $\kappa_{j}$ \\
\hline$C_{1}$ & 1 & 2 & 0 & 0 & 0 & 0 \\
\hline$C_{2}$ & 5 & 6 & 0 & 0 & 0 & 0 \\
\hline$C_{3}^{(1)}$ & 9 & 11 & 0 & 0 & 0 & 0 \\
\hline$C_{3}^{(2)}$ & 9 & 10 & 0 & 0 & 0 & 0 \\
\hline$C_{4}$ & 12 & 13 & 1 & 0 & $\frac{\pi}{2}$ & 0 \\
\hline$C_{5}$ & 14 & 15 & 0 & 0 & 0 & 0 \\
\hline$C_{6}$ & 16 & $\emptyset$ & 0 & 0 & 0 & 0 \\
\hline$C_{7}$ & 3 & 4 & 0 & 0 & 0 & 0 \\
\hline$C_{8}$ & 7 & 8 & 0 & 0 & 0 & 0 \\
\hline
\end{tabular}

\subsubsection{Stiffness matrix}

$H=17$ for the FANUC S420iF. So ${ }^{0} \mathbf{K}_{c}$ is a $(102 \times 102)$ square matrix. Then, boundary conditions are introduced to express the fact that the robot is embedded to the ground at the node 0 . The size of stiffness matrix is then reduced by deleting rows and 
columns corresponding to the nodes for which displacements are null. The dimension of ${ }^{0} \mathbf{K}_{c}^{*}$ is $(96 \times 96)$.

\subsubsection{Rigid link motion}

There are $n=8$ links for the FANUC S420iF. Then $M=6 \times(8-1)=42$ and the dimension of ${ }^{0} \mathbf{A}_{c}^{*}$ is $(42 \times 96)$.

\subsubsection{Equivalent structure}

With the relation (15), all node displacements are known. In this paper only the displacement of the node 16 is necessary. It represents the end-effector of the robot. In the case of the FANUC S420iF, relation (13) becomes:

$$
\left[\begin{array}{cc|c}
{ }^{0} \mathbf{K}_{c(90,90)}^{*}{ }^{0} \mathbf{A}_{c(90,42)}^{* T} & { }^{0} \mathbf{K}_{c(90,6)}^{*} \\
{ }^{0} \mathbf{A}_{c(42,90)}^{*} & \mathbf{0}_{(42,42)} & { }^{0} \mathbf{A}_{c(42,6)}^{*} \\
\hline{ }^{0} \mathbf{K}_{c(6,90)}^{*}{ }^{0} \mathbf{A}_{c(6,42)}^{* T} & { }^{0} \mathbf{K}_{c(6,6)}^{*}
\end{array}\right]\left[\begin{array}{c}
{ }^{0} \Delta \mathbf{X}_{c(90,1)}^{*} \\
\frac{\lambda_{(42,1)}}{{ }^{0} \Delta \mathbf{X}_{c(6,1)}^{*}}
\end{array}\right]=\left[\begin{array}{c}
{ }^{0} \mathbf{F}_{c(90,1)}^{*} \\
\frac{\mathbf{0}(92,1)}{{ }^{0} \mathbf{F}_{c(6,1)}^{*}}
\end{array}\right] .
$$

By applying equation (19), the elastic model is obtained:

$$
{ }^{0} \Delta \mathbf{X}_{c(6,1)}^{*}={ }^{0} \Delta \mathbf{X}_{E}=\left({ }^{0} \mathbf{K}_{e q}\right){ }^{-1}{ }^{0} \mathbf{F}_{e q} .
$$

\subsection{Elastic identification}

Elastic calibration consists in identifying the stiffness parameters of joints and the geometrical characteristics of $C_{4}$. This is done independently of the geometrical errors whose effects on the elastic behavior are considered as negligible. The experimental setup is shown on Figure 6. It has been applied onto the FANUC robot by using the Nikon Metrology K600-10 photogrammetric measurement system for a precise tracking of the moving end-effector. TCP poses have been generated over the workspace of the FANUC S420iF. A complete characterization of the robot has been obtained by stressing all its joints. For that purpose, a cable-pulley device is used to generate forces at the end-effector along all axis of the reference frame $R_{0}$. The direction of the force $\mathbf{F}_{2}$ is measured by an LED fixed directly on the cable (Figure 6). Moreover, a fixture is connected to the end-point to apply a higher force $\mathbf{F}_{1}$ along $\mathbf{z}_{0}$ axis. Finally a grid of 150 poses is defined, with two load configurations:

- $800 N$ along $\mathbf{F}_{\mathbf{1}}$, and $600 N$ along $\mathbf{F}_{2}$

- $600 N$ along $\mathbf{F}_{1}$, and $200 N$ along $\mathbf{F}_{2}$

The first load is used for elastic identification because it fits a typical force case in the ISF application. The second load is used for model verification.

For each joint, four stiffness parameters have to be identified, with the assumption that the two radial and axial translational stiffness are equal. For the passive joints 3, 8 and $9, K_{a r}$ cannot be null for numerical problems, so it has been chosen to set this parameter to $10^{-1} \mathrm{Nmm} . \mathrm{rad}^{-1}$. The other stiffness values have been identified from measurements performed directly on the robot. For the FANUC S420iF, 33 joint stiffness values have to be identified from measured data. The differences between controlled and reached poses have been measured for each level of payload and without to obtain the real elastic displacements. If ${ }^{0} \Delta \mathbf{X}_{E}^{m, p}$ and ${ }^{0} \Delta \mathbf{X}_{E}^{p}$ stand respectively for the vectors of the measured and calculated displacements for the pose and the load $p$, the error that is used to identify the structure elastic model is:

$$
{ }^{0} E_{E}^{p}=\left\|{ }^{0} \Delta \mathbf{P}_{E}^{p}-{ }^{0} \Delta \mathbf{P}_{E}^{m, p}\right\| .
$$




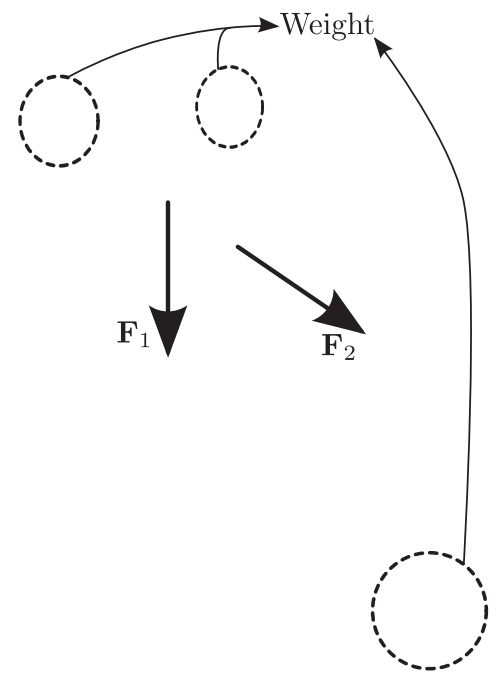

Figure 6: Experimental setup

The numerical values of the joint stiffness values gathered in the vector $\Gamma$ are identified by minimizing, for a set of $n_{m}$ poses and loads, the following criterium:

$$
C(\Gamma)=\sum_{i=1}^{n_{m}} \sqrt{\left({ }^{0} E_{E}^{p}\right)^{2}} .
$$

To identify the stiffness parameters, an optimization procedure is implemented in modeFRONTIER, a multi-objective optimization software, using a genetic optimization algorithm modeFRONTIER, ESTECO srl-EnginSoft ${ }^{\odot}$ [27]. An object-oriented programming language, MATLAB $2008 \mathrm{~b}^{\odot}$, is used for the modeling. The identified stiffness values obtained with the optimum design are used to evaluate the elastic displacements of the robot for the verification load configuration. The results for horizontal, lateral and vertical displacements are given in Figure 7. When the robot is far of $\mathbf{z}_{0}$ and the ground, the elastic displacements are more important. On the contrary, the stiffness of the robot increases near of $\mathbf{z}_{0}$ and the ground. The model gives a good prediction of the elastic behavior with a maximum TCP pose error of $\pm 0.35 \mathrm{~mm}$ and a mean error about $\pm 0.15 \mathrm{~mm}$ over the workspace. This is compatible with ISF requirements within a prototyping context [23]. 

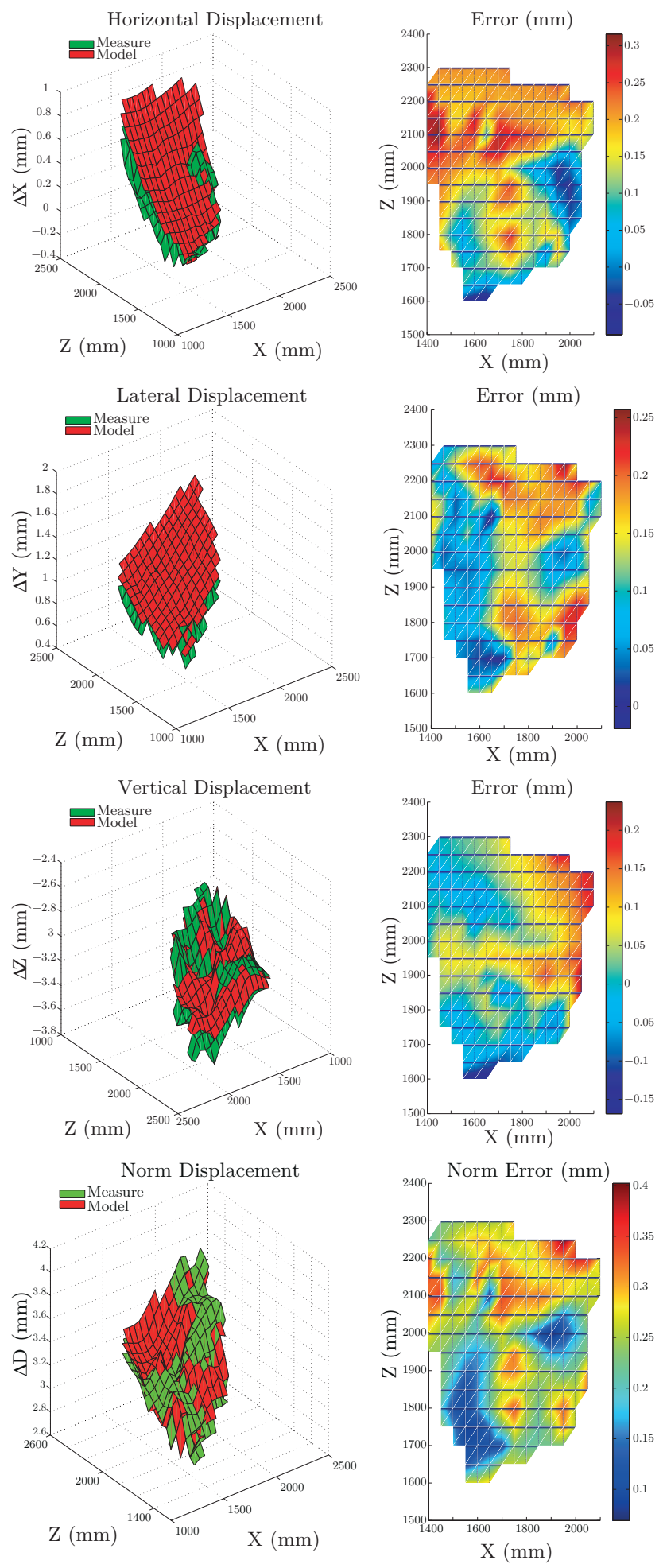

Figure 7: Results for load $600 N$ along $\mathbf{F}_{1}-200 N$ along $\mathbf{F}_{2}$ 


\section{Experimental validation: application to ISF process}

A post-processor including the $\mathrm{FE}$ analysis of the process and the elastic model of the FANUC S420iF is developed (Figure 8) as proposed in [20].

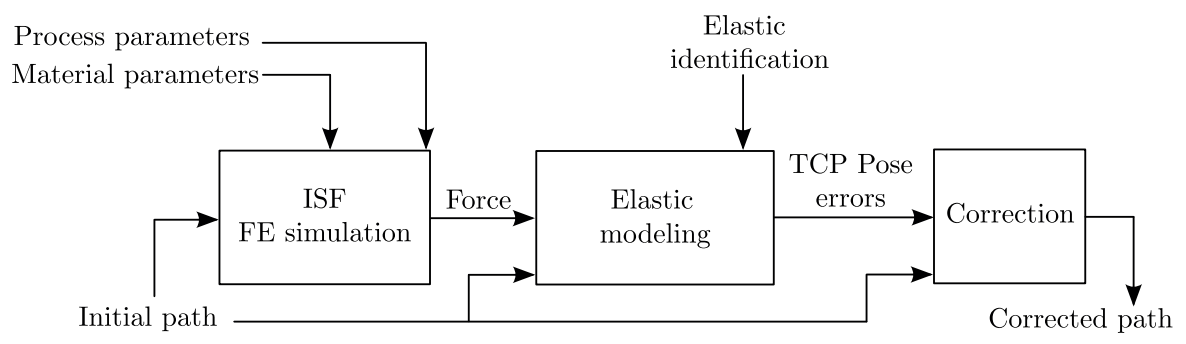

Figure 8: Post-processor scheme

The process FE simulation performed with the ABAQUS ${ }^{\odot}$ software computes the estimated forces required to form the part assuming an ideal stiff robot structure. The advantage of this approach is to avoid the measuring of the forming forces during a first run without any compensation as in [20]. Actually in the case of really compliant robots, the measuring forces must be really lower as those exerted by an assuming stiff structure and this can lead to inaccurate corrections of the tool path. This effect was verified by forming without applying any compensation a frustum cone (Figure 10) with the Fanuc S420if robot and a FAMUP MCX500 three axes cartesian milling machine. This machine can developed up to $7000 \mathrm{~N}$ at the end of the tool with a pose accuracy of $15 \mu \mathrm{m}$ and therefore the errors on the tool path induced by its elastic deformations can be assumed as neglectable. As one can see on Figure 9, the difference between the mean forces measured along the tool axis on the miling machine and the robot is about $35 \%$. This shows that taking into account the forming forces measured during a first run with the robot will obviously result in inaccurate corrections of the tool path.
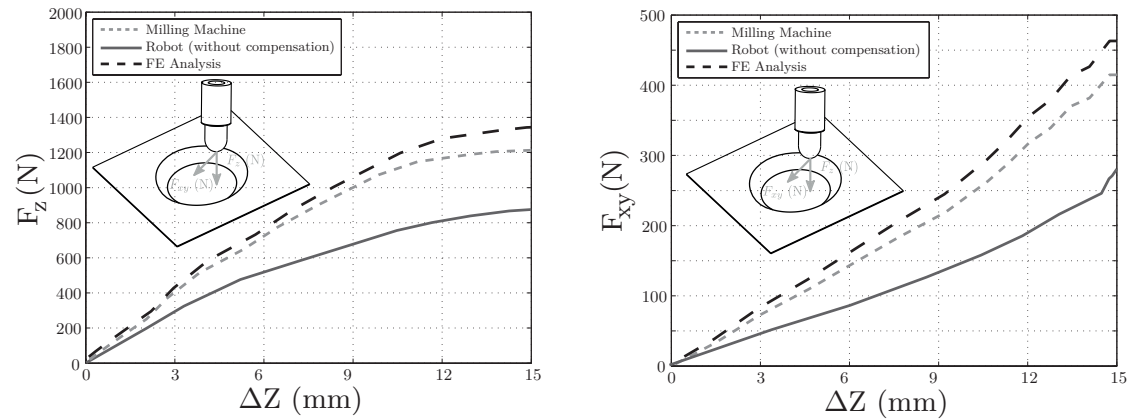

Figure 9: Comparison of the measured and simulated curves of the mean forces $\left(F_{z}\right.$ and $\left.F_{x y}\right)$ during the forming of a frustum cone

To compute accurately the estimated forces with the FE simulation performed on ABAQUS ${ }^{\odot}$, the influence of three factors has been particulary investigated:

- The through-thickness shear: the deformation mechanisms of the process are not well identified [28] but some authors have shown that the through-thickness 
shear must be considered during the process [29]. The influence of this phenomenon on the predicted force has been quantified by comparing two types of elements (brick (C3D8I) and shell (S4R) elements of ABAQUS ${ }^{\odot}$ software).

- The hardening law: the high level of deformation reached by the process, implies investigations on the choice of the hardening law. Hardening laws are typically identified with tensile test until a low level of strain (about 20\%). It can result in an approximation of the force needed by the ISF process for high levels of strain (between $60 \%$ and $80 \%$ ) [30]. Two formulations, identified by the same experimental setup, have been implemented in the simulation software. The first law is the classical power Ludwick law. The second is a Voce Law, often used to model saturation or material softening especially for aluminium alloys.

- The modeling of the clamping system: encastre boundary condition can lead to an artificial stiffening of the model [31]. However, sliding can appear between the sheet and the clamping system during the forming process. This hypothesis was verified comparing a stiff model and a more realistic model including the clamping pressure.

To verify the efficiency of the FE analysis, the most accurate predicted force (obtained with C3D8I elements, a Voce Law and a realistic modeling of the clamping pressure) along the tool axis has been compared to the one measured directly on the milling machine. Figure 9 shows the good qualitative agreement (less than $10 \%$ error) between the predicted and the measured forces. This shows that the simulated force can be taken as a reference value to calculate accurately the TCP pose errors induced by the elastic deformations of the robot structure with the proposed elastic model. And then those errors can be added to the nominal path to obtain the final corrected tool path.

\subsection{Frustum cone}

In order to check the whole procedure validity and to estimate the resulting forming errors, the same frustum cone as previously is used. The $3 \mathrm{D}$ complex path used to form it is defined in the user frame $\mathbf{R}_{p}=\left(\mathbf{O}_{p}, \mathbf{x}_{p}, \mathbf{y}_{p}, \mathbf{z}_{p}\right)$. The cone of $45^{\circ}$ wall angle is centered on a sheet of $200 \times 200 \times 1 \mathrm{~mm}^{3}$. The infeed direction is along $\mathbf{z}_{p}$ but the tool trajectory stresses the robot structure in all directions (Figure 10). The material is an Al $5086 \mathrm{H} 111$ and its mechanical behavior has been already studied in a previous paper [32]. The tool diameter is $15 \mathrm{~mm}$ and, in order to minimize friction, grease is used. The wrenches apply by the tool are measured by a six-axis force/torque sensor ATI Omega190. During the forming process the real tool path is measured by the Nikon Metrology K600-10 photogrammetric measurement system.

The error between the target and measured tool paths before and after correction are depicted in Figure 11. For more legibility, the error along $\mathbf{x}_{p}$ (Figure 11(a)), $\mathbf{y}_{p}$ (Figure 11(b)), $\mathbf{z}_{p}$ (Figure 11(c)) and the norm error (Figure 11(d)) are presented separately in the plane $\left(\mathbf{O}_{p}, \mathbf{x}_{p}, \mathbf{y}_{p}\right)$ for the whole tool path. As one can see :

- Without correction: a significant TCP deviation can be remarked. The maximum errors are about $-2.5 \mathrm{~mm}, \pm 1.5 \mathrm{~mm}$ and $-3.5 \mathrm{~mm}$ respectively along $\mathbf{x}_{p}, \mathbf{y}_{p}$ and $\mathbf{z}_{p}$ and the absolute values of the mean errors are about $0.8 \mathrm{~mm}, 0.45$ $\mathrm{mm}$ and $1.2 \mathrm{~mm}$. The maximum value of the error norm is about $4 \mathrm{~mm}$ at the end of the trajectory and the mean value is about $1.8 \mathrm{~mm}$. 

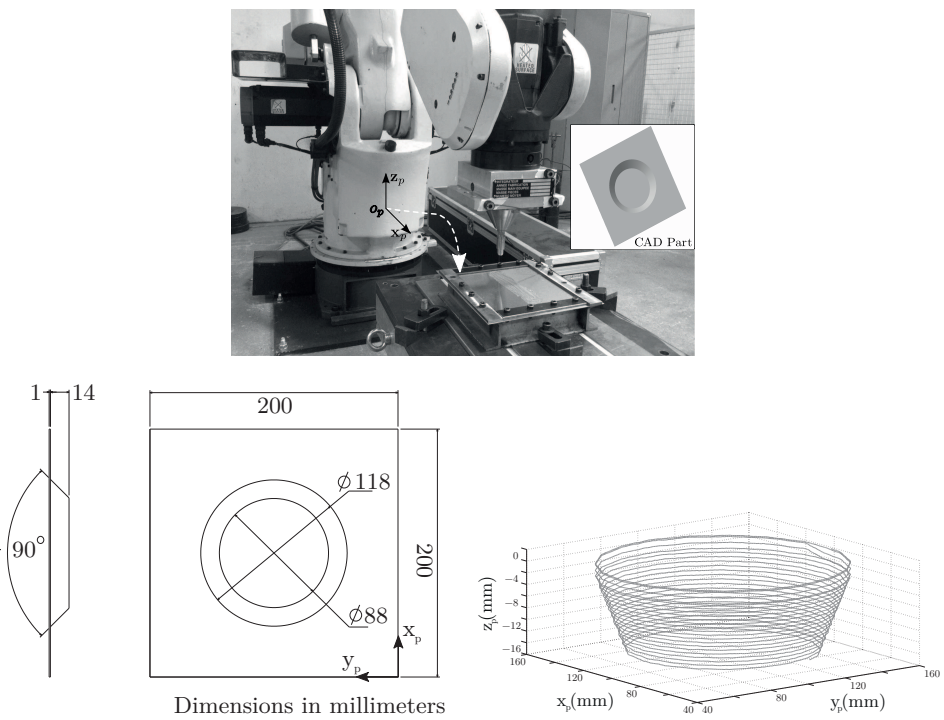

Figure 10: Experimental setup and target tool path of the frustum cone

The Figure 11(a) shows that the geometrical error along $\mathbf{x}_{p}$ is not uniformly distributed along the path. It leads to a concentricity defect of $3.2 \mathrm{~mm}$ (Figure 12) which value has been calculated with the least square method by considering the target and measured last circles of each trajectory. This defect is related to the direction of the resulting forces. The Figure 13 shows the robot joint configurations when the tool is on points 1 and 2 corresponding to the minimum and maximum positions along $\mathbf{x}_{p}$ (Figure 12). For a given configuration the vector of the torques $\boldsymbol{\Gamma}_{e}$ that are exerted by the robot joints can be calculated according to the relation:

$$
\boldsymbol{\Gamma}_{e}=-{ }^{E} \mathbf{J}_{E}{ }^{T}{ }^{E} \mathbf{F}_{e}
$$

where ${ }^{E} \mathbf{J}_{E}$ is the jacobian matrix of the robot and ${ }^{E} \mathbf{F}_{e}=\left[F_{x}, F_{y}, F_{z}, M_{x}, M_{y}\right.$, $\left.M_{z}\right]^{T}$ is the vector of the resulting forces due to the environment on the TCP robot.

The resulting force $F_{x y}$ in the plane of the metal sheet can be calculated by :

$$
F_{x y}=\sqrt{F_{x}^{2}+F_{y}^{2}}
$$

The direction of this force depends on the TCP position along the tool path. For minimum and maximum values of $\mathbf{x}_{p}$, the force is along $\mathbf{x}_{p}$ axis but with opposite directions. By verifying the relation (28), one can see on the Figure 13 that the torques $M_{3, F_{z}}$ and $M_{5, F_{z}}$ resulting of the application of the force $F_{z}$ on joints 3 and 5 respectively act always on the same sense. At the contrary, the torques $M_{3, F_{x y}}$ and $M_{5, F_{x y}}$ related to the force $F_{x y}$ can act either on the same (point 1) or on the opposite sense (point 2). This is shown on Figure 13. As a consequence, the TCP elastic displacements are higher at the neighborhood of point 1 than they are at the point 2 despite the robot structure is stiffer. 


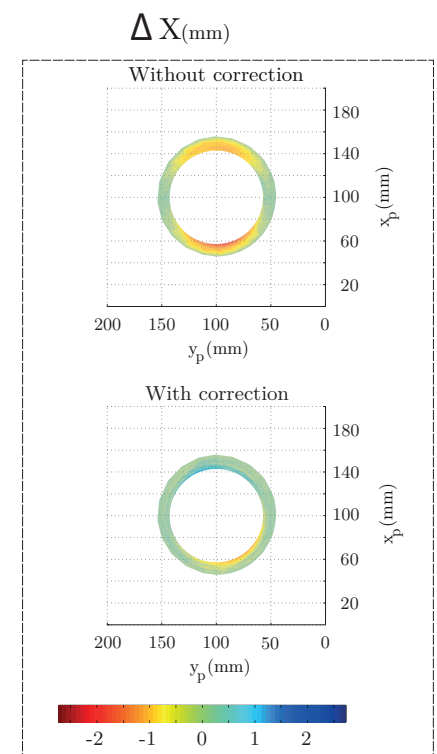

(a)

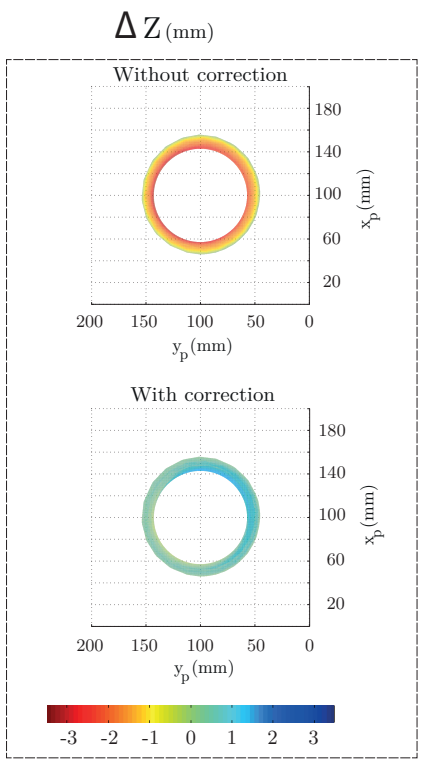

(c)

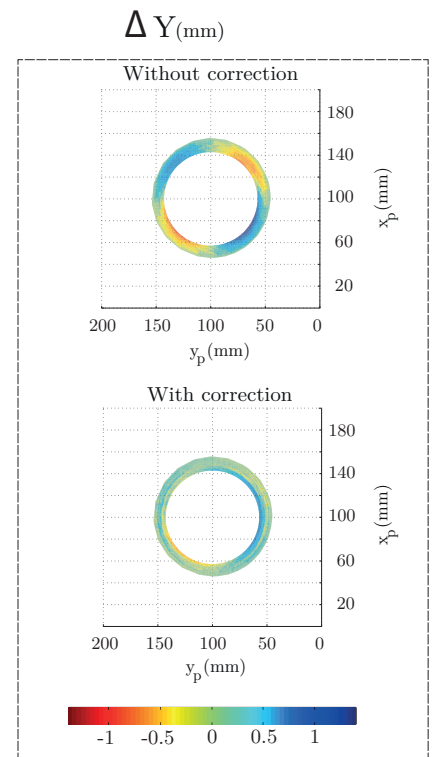

(b)

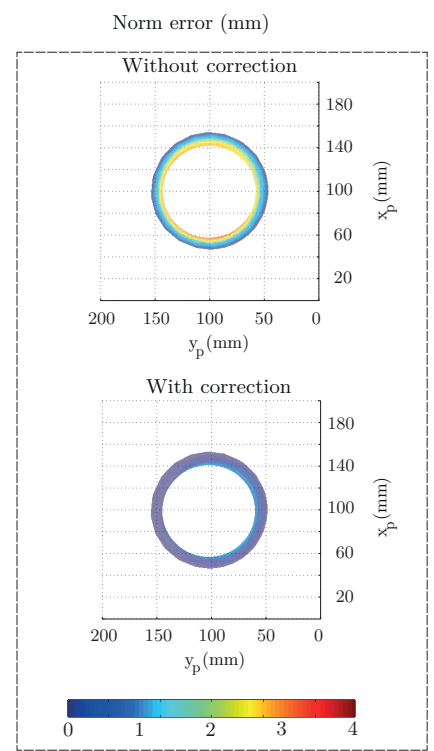

(d)

Figure 11: Tool path errors along $\mathbf{x}_{p}$ (a), $\mathbf{y}_{p}$ (b), $\mathbf{z}_{p}$ (c) and the norm error (d) (without and with correction) during the forming of the frustum cone

Figure 11(b) shows that $\Delta Y$ is symmetric with respect to the center of the tool path. This is due to the fact that there is no combination of the torques on the robot joints as explained before. This error increases in function of the tool position along $\mathbf{z}_{p}$. This phenomenon is due to the evolution of the force $F_{x y}$ during the tool path (Figure 9). 
$F_{z}$ presents the same evolution than $F_{x y}$ (Figure 9). As a consequence $\Delta Z$ increases as a function of the infeed value along $\mathbf{z}_{p}$ (Figure 11(c)).

Obviously these errors are not compatible with the process requirements. That is why the trajectory is corrected using the off-line post-processor previously described.
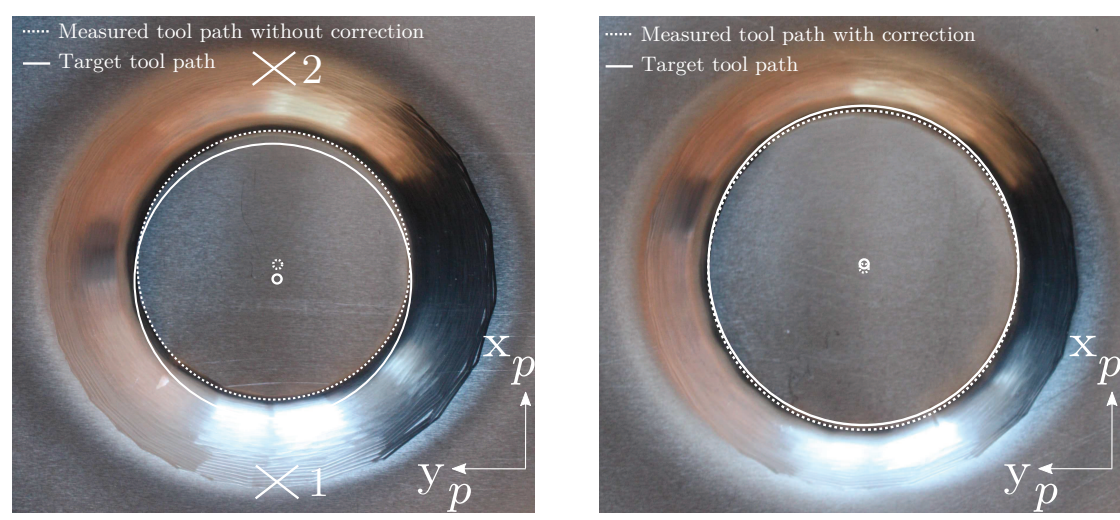

Figure 12: Part pictures - Comparison of concentricity defects
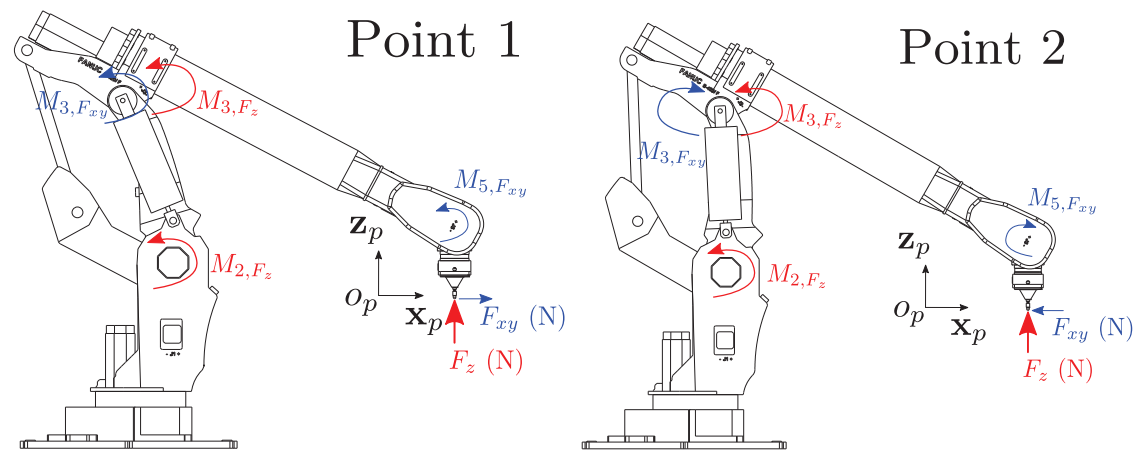

Figure 13: Analyse of motor torque and environment forces

- With correction: a significant improvement is obtained. Maximum errors are about $-0.9 \mathrm{~mm}, \pm 1 \mathrm{~mm}$ and $1 \mathrm{~mm}$ respectively along $\mathbf{x}_{p}, \mathbf{y}_{p}$ and $\mathbf{z}_{p}$. Absolute values of mean errors are about $0.15 \mathrm{~mm}, 0.14 \mathrm{~mm}$ and $0.22 \mathrm{~mm}$ along the same axes. The maximum value of the error norm is about $1 \mathrm{~mm}$ at the end of the trajectory and the mean value is about $0.25 \mathrm{~mm}$. The concentricity defect value is reduced to $0.6 \mathrm{~mm}$. The remaining errors are mainly due to the differences between the simulated and measured forces (Figure 9). They increase along the trajectory and induce some maximum errors at the end of the tool path. This effect is particularly visible with $\Delta Z$. After correction with the proposed postprocessor the maximum error $\Delta Z$ is about $1 \mathrm{~mm}$ against $-3.5 \mathrm{~mm}$ without correction. It is due to the overestimation of the force $F_{z}$ which leads to an overcorrection of the tool path along $\mathbf{z}_{p}$. 
The first obtained experimental results show the method relevance. The TCP pose accuracy is improved of $70 \%$ during the forming of the frustum cone. The trajectory concentricity is also increased of $80 \%$. This validate the process requirements.

\subsection{Twisted pyramid}

The other part that is formed to confirm the previous results is a twisted pyramid made in the same material as the frustum cone. Its non-symetrical geometry allows to validate the robustness of both the process FE analysis and the elasto geometrical calibration of the FANUC robot (Figure 14). The trajectory consists in constant $\mathrm{z}$ levels (Figure 14) with an infeed value $\Delta_{z}$ is $1 \mathrm{~mm}$ per loop. The force $F_{z}$ is measured by strain gauges sensor fixed on the tool.
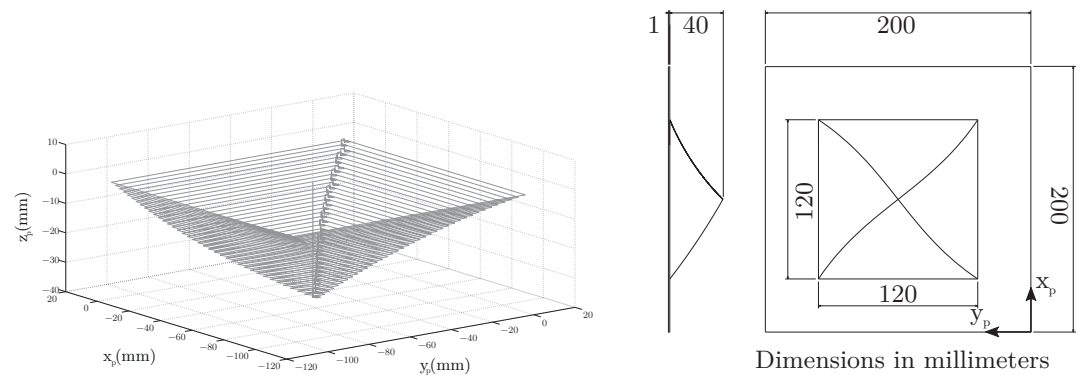

Figure 14: Shape and target tool path of the twisted pyramid

The absolute errors between the target and measured tool paths before and after correction given in the user frame $R_{p}$ are depicted in Figure 15. The following conclusions can be deduced:

- Without correction: A significant TCP deviation can be observed. The maximum value of the error norm is about $6 \mathrm{~mm}$ at the end of the trajectory and the mean value is about $3 \mathrm{~mm}$. The non-symmetry of the part implies the non-symmetry of the error. This phenomenon is explained by the changing of inclination of the four faces of the twisted pyramid. It leads to change the direction of $F_{x}$ during the path that has some consequences on the torques values $M_{3, F_{x y}}$ and $M_{5, F_{x y}}$ as explained before (Figure 13).

- With correction: An important improvement of the pose accuracy is obtained. The maximum value of the error norm is about $1 \mathrm{~mm}$ and the mean value is about $0.6 \mathrm{~mm}$. The effect of the inclination of each faces of the pyramid is well compensated thanks to the good prediction of the forming forces by the FE simulation (Figure 16) as already shown in section 5 and to the other hand to the realistic identification of the elastic behavior of the FANUC robot structure. The final TCP error that can be observed after the tool path compensation is mainly induced by the residual identification errors remaining after the elastic calibration. They introduce a difference between the predicted and measured forces which grows up with the infeed value (Figure 16). However, even considering these errors, the TCP pose accuracy can be improved about $80 \%$ during the forming of this part. This allows the final geometry of the part to converge towards the nominal specifications required by a prototyping application. 
Norm Error (mm)

Without correction

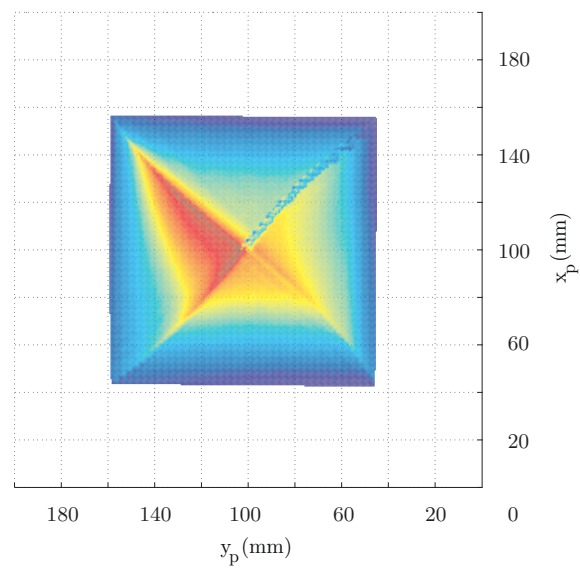

With correction

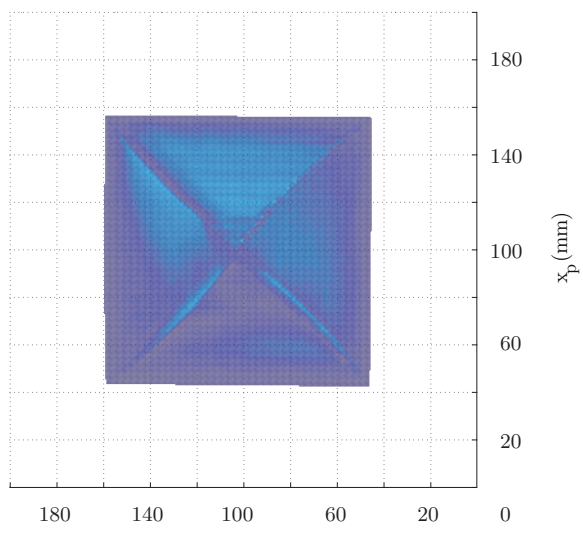

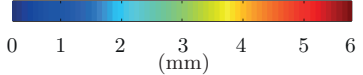

Figure 15: Error norm measured between the target and measured tool paths (without and with correction) during the forming of the twisted pyramid

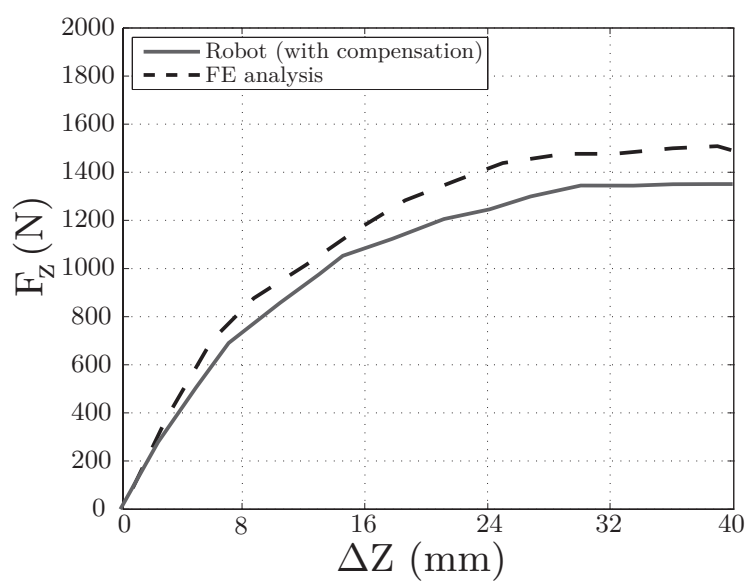

Figure 16: Measured and predicted forces $\left(F_{z}\right)$ of the twisted pyramid

\section{CONCLUSION}

This paper brings a contribution to the works of Meier and al. [20] [1]. First, instead of a MSB modeling, a robust and systematic notation is proposed for the elastic modeling of industrial open- and closed-loop robot manipulators. Second, due to an efficient FE analysis of the forming task, the predicted forming forces are computed and then used as the inputs of the proposed elastic model. This avoids to measure the forming forces during a first run without any compensation as in [20]. The proposed contributions have been involved to plan the trajectories of a FANUC robot used in forming applications to fully compensate the TCP pose errors due to the robot elastic 
deformations. The obtained experimental results show the relevance of this approach since the TCP pose accuracy could be improved up to $80 \%$ for the forming of a frustum cone and a non-symmetrical part (a twisted pyramid) made in an aluminium sheets. This allows the final geometry of the formed parts to converge towards the nominal specifications required by a prototyping application.

\section{References}

[1] H. Meier, B. Buff, R. Laurischkat, V. Smukala, Increasing the part accuracy in dieless robot-based incremental sheet metal forming, CIRP Annals - Manufacturing Technology 58 (2009) 233-238.

[2] O. Marcotte, L. Vanden-Abeele, $2 \mathrm{~d}$ and $3 \mathrm{~d}$ friction stir welding with articulated robot arm, 8th International FSW Symposium (2010) 1-20.

[3] J. Tuomi, L. Vihtonen, Incremental sheet forming as rapid prototyping and manufacturing technology, International Conference on Manufacturing Automation (2007).

[4] J. L. Caenen, J. C. Angue, Identification of geometric and non-geometric parameters of robots, IEEE International Conference on Robotics and Automation 2 (1990) 1032-1037.

[5] K. Schroer, S. L. Albright, A. Lisounkin, Modeling closed-loop mechanisms in robots for purposes of calibration, IEEE Transactions on Robotics and Automation 13 (1997) 218-229.

[6] J. Denavit, R. S. Hartenberg, A kinematic notation for a lower-pair mechanisms based on matrices, ASME Journal of Applied Mechanics 22 (1955) 215-221.

[7] S. Hayati, Robot arm geometrical link parameter estimation, IEEE International Conference on Decision and Control, San Antonio, Texas, USA (1983) 14771483.

[8] W. Khalil, E. Dombre, Modeling, Identification and Controls of Robots, Hermes Penton Ltd, 2nd edition, 2002.

[9] M. W. Spong, K. Khorasani, P. V. Kokotovic, An integral manifold approach to the feedback control of flexible joint robots, IEEE Journal of Robotics and Automation 3 (1987) 291-300.

[10] W. Khalil, M. Gautier, Modeling of mechanical systems with lumped elasticity, IEEE International Conference on Robotics and Automation, San Francisco, CA, USA 4 (2000) 3964-3969.

[11] A. Bres, B. Monsarrat, L. Dubourg, L. Birglen, C. Perron, M. Jahazi, L. Baron, Simulation of robotic friction stir welding of aerospace components, Industrial Robot: An International Journal 37 (2010) 36-50.

[12] P. Bigras, M. Lambert, C. Perron, New formulation for an industrial robot force controller: Real-time implementation on a kuka robot, IEEE International Conference on Systems, Man and Cybernetics (2007) 2794-2799. 
[13] C. C. de Wit, G. Bastin, B. Siciliano, Theory of Robot Control, Springer-Verlag New York, Inc., Secaucus, NJ, USA, 1st edition, 1996.

[14] C. Gosselin, D. Zhuang, Stiffness analysis of parallel mechanisms using a lumped model, International Journal of Robotics and Automation 17 (2002) 17-27.

[15] F. Majou, C. Gosselin, P. Wenger, D. Chablat, Parametric stiffness analysis of the orthoglide, Mechanism and Machine Theory 42 (2007) 296-311.

[16] C. Dumas, S. Caro, S. Garnier, B. Furet, Joint stiffness identification of sixrevolute industrial serial robots, Robotics and Computer-Integrated Manufacturing 27 (2011) 881-888.

[17] D. Deblaise, X. Hernot, P. Maurine, A systematic analytical method for pkm stiffness matrix calculation, IEEE International Conference on Robotics and Automation, Orlando, Florida, USA (2006) 4213-4219.

[18] S. Marie, P. Maurine, Elasto-geometrical modelling of closed-loop industrial robots used for machining applications, IEEE International Conference on Robotics and Automation (2008) 1294-1300.

[19] G. Ecorchard, R. Neugebauer, P. Maurine, Elasto-geometrical modeling and calibration of redundantly actuated pkms, Mechanism and Machine Theory 45 (2010) 795-810.

[20] H. Meier, R. Laurischkat, J. Zhu, A model based approach to increase the part accuracy in robot based incremental sheet metal forming, AIP Conference Proceedings 1315 (2011) 1407-1412.

[21] J. Duflou, Y. Tunçkol, A. Szekeres, P. Vanherck, Experimental study on force measurements for single point incremental forming, Journal of Materials Processing Technology 189 (2007) 65-72.

[22] N. F. ISO-9283, Robots manipulateurs industriels - critères de performance et méthodes d'essai correspondantes (1998).

[23] J. Jeswiet, F. Micari, G. Hirt, A. Bramley, J. Duflou, J. Allwood, Asymmetric single point incremental forming of sheet metal, CIRP Annals - Manufacturing Technology 54 (2005) 88-114.

[24] J. F. Imbert, Analyse des structures par éléments finis, Cepadues Edition, Toulouse, France, 1979.

[25] W. Khalil, J. F. Kleinfinger, A new geometric notation for open and closed loop robots, IEEE International Conference on Robotics and Automation, San Francisco, California, USA 2 (1986) 1174-1180.

[26] W. K. Yoon, T. Suehiro, Y. Tsumaki, M. Uchiyama, Stiffness analysis and design of a compact modified delta parallel mechanism, Robotica 22 (2004) 463-475.

[27] E. Courteille, D. Deblaise, P. Maurine, Design optimization of a delta-like parallel robot through global stiffness performance evaluation, IEEE/RSJ International Conference on Intelligent Robots and Systems (2009) 5159-5166. 
[28] W. Emmens, A. van den Boogaard, An overview of stabilizing deformation mechanisms in incremental sheet forming, Journal of Materials Processing Technology 209 (2009) 3688-3695.

[29] P. Eyckens, J. Del-lero Moreau, J. Duflou, A. Van Bael, P. Van Hoote, MK Modelling of sheet formability in the incremental sheet forming process, taking into-account through-thickness shear, International Journal of Material Forming (2009) 379-382.

[30] C. Henrard, C. Bouffioux, P. Eyckens, H. Sol, J. Duflou, A. Van Bael, P. Van Houte, L. Duchêne, A. Habraken, Forming forces in single point incremental forming: prediction by finite element simulations, validation and sensivity, Computational mechanics 47 (2011) 573-590.

[31] C. Bouffioux, C. Henrard, J. Gu, J. Duflou, A. Habraken, H. Sol, Development of an inverse method for identification of materials parameters in the single point incremental forming process, IDDRG 2007 (2007).

[32] C. Zhang, L. Leotoing, D. Guines, E. Ragneau, Experimental and numerical study on effect of forming rate on AA5086 sheet formability, Materials Science and Engineering A 527 (2010) 967-972. 